\title{
Prevalence of Intestinal Parasitic Infections during Upper Gastrointestinal Endoscopy
}

\author{
Noha Essam Eldin Abd El Rehem Shalaby ${ }^{1 *}$, Ahmad Ali Othman ${ }^{1}$, \\ Mamdouh Ahmed Gabr ${ }^{2}$ and Nabila Abd El Azeem Yassen ${ }^{1}$ \\ ${ }^{1}$ Medical Parasitology, Internal Medicine, ${ }^{2}$ Departments, Faculty of Medicine, \\ Tanta University, Egypt \\ *Corresponding author
}

\section{Keywords \\ Intestinal parasitic infections, Upper gastrointestinal endoscopy, Giardia duodenalis and Cryptosporidium parvum}

Article Info

Accepted:

22 June 2020

Available Online:

10 July 2020

\section{A B S T R A C T}

Helminthic and protozoal infections of gastrointestinal tract remain a major health problem worldwide especially in tropical and subtropical areas. Light microscopy of stool specimens is the most frequently used for diagnosis of parasitic infections. However, it is time-consuming, and requires experienced staff. The aim of this study was to evaluate the frequency of parasitic infections during routine upper gastrointestinal endoscopy. Patients with different age groups admitted for upper endoscopy for any indication were subjected to informed consent, questionnaire including name, age, gender, residence, and indication for endoscopy. Duodenal aspirate samples were examined microscopically by direct smear examination, iodine, modified Ziehl-Neelsen stain, and immunodiagnostic technique for detection of Cryptosporidium and Giardia antigens. Histopathological examination of duodenal biopsies by hematoxylin and eosin stain for detection of parasites was also performed. This study was conducted on 70 patients. Most of parasitic infections were more prevalent in males than females, especially in age group from (40-60) years. Giardia duodenalis and Cryptosporidium parvum were the most prevalent parasites. Cyclospora cayetanensis and Entamoeba histolytica were also detected. Ascaris lumbricoides was identified in one case by direct visualization by endoscope. Iodine and modified ZiehlNeelsen stains for diagnosis of Giardia and Cryptosporidium respectively were more accurate than direct smear examination and rapid immunoassay test. Histopathological examination of duodenal biopsies had detected parasites and their pathological features of inflammation. Upper gastrointestinal endoscopy proved a useful tool in the diagnosis of parasitic infections.

\section{Introduction}

Gastrointestinal parasitosis is a significant cause of morbidity and mortality. Although it is particularly more common in underdeveloped regions with poor sanitary conditions, they are present throughout the whole world. Clinically, it may vary depending on the parasite type and the affected regions of gastrointestinal tract (GIT) 
(Ali et al., 2008).Usually, the diagnosis of alimentary tract parasites is made by characteristic findings as eosinophilia and egg shape appearance on examination of fecal samples (Mohamed et al., 2000). However, misdiagnosis may be due to absence of eggs in stool. Moreover, there are some reports of parasitic diagnosis during routine upper endoscopy (Zaher et al., 2012).

Intestinal protozoa are particularly important due to difficulty in their diagnosis. As Giardia cysts can be excreted intermittently, many cases $(>50 \%)$ of giardiasis will be missed. Therefore, when giardiasis is suspected and stool specimens are negative, the string test, duodenal aspiration, or biopsy can be performed.

In a fresh specimen, trophozoites usually can be visualized on direct wet mount. Moreover, in Cryptosporidium parvum infection, oocyst shedding in feces can be intermittent, and several fecal specimens (at least three for an immunocompetent host) should be collected for microscopic examination (Washam and Frenck, 2018).

Some cases may require tissue evaluation in order to rule out other GIT pathologies. Although pathologists are usually familiar with common parasites, there is no adequate knowledge about direct microscopic visualization of parasites in biopsy samples (Gupta et al., 2009).

In addition, it is not known whether there are specific clues to suspect a parasitic infection or not, especially in cases where the microorganism is either very sparse or not visualized on small biopsy material or when there is no relevant clinical information upon the possibility of a parasitic infection (Pehlivanoğlu et al., 2016).

Obtaining of duodenal fluid during the upper gastrointestinal endoscopy and microscopic examination of it can help in the diagnosis of parasites like Giardia lamblia especially in patients with persistent diarrhea. Also intestinal aspirates from immunocompromised patients may show protozoa: Giardia, Cystoisospora, Cryptosporidia, and helminths as Strongyloides stercoralis and Fasciola (Wahnschaffe et al., 2007; Bhaijee et al., 2011).

The biopsy sample can be used to make touch preparations for identifying Giardia in tissue sections and for histological examination. In addition, Cryptosporidium parvum can be found along the microvillus region of the epithelia that line the gastrointestinal tract. Histolopathological examination may reveal villous atrophy and blunting, and inflammation of the lamina propria (Robert et al., 2016).

This study aimed to determine the frequency of intestinal parasitic infections among patients undergoing upper gastrointestinal endoscopic procedures in the endoscopy units of Tanta University Hospitals.

\section{Materials and Methods}

\section{Subjects}

In the period from May 2018 to July 2019, a total of 70 patients underwent upper gastrointestinal (GI) endoscopy for various upper GI symptoms. They were subject of the present study. They were randomly selected from Endoscopy unit, Tanta University.

\section{Inclusion criteria}

The study enrolled patients of different age groups with different clinical presentations who were admitted for upper gastrointestinal endoscopy during one year from the start of the study. 
For conduction of this study all patients were subjected to the following

Informed consent was obtained from all participants.

Interviewing questionnaire including demographic data such as age, gender, name, level of education, occupation, housing conditions regarding crowding index and source of water; clinical data such as symptoms of abdominal colic, vomiting, diarrhea and also the indication for endoscopy.

Technique of upper gastrointestinal endoscopy (Koch and Zurad, 2020)

Duodenal aspirate samples were collected from the second part of the duodenum through a polythene tube passed via the biopsy channel. The polythene tube was specially prepared with multiple perforations on the sides. A sterile syringe was attached to the polythene tube and duodenal juice collected in a clean tight fitting container. Then every sample was labeled properly with the patient's name.

Biopsies were taken from the duodenal mucosa using biopsy forceps.

\section{Parasitological study}

Duodenal aspiration samples (El-Hady et al., 2018)

After performing concentration by centrifugation of the fluid at $500 \times \mathrm{g}$ for $5 \mathrm{~min}$ and the supernatant was decanted. Microscopic examinationwas done on the sediment as direct wet mount as well as after staining the samples with Lugol's iodine and modified Ziehl- Neelsen stain for detection of oocysts of coccidian.

\section{Direct smear examination (Carleton et al., 1980)}

Saline wet mount is used for the detection of trophozoites and cysts of protozoa, also it is used for detection of eggs and larvae of helminths.

Modified Ziehl-Neelsen stain (Rosenblatt $\boldsymbol{e t}$ al., 2009)

The modified Ziehl-Neelsen stain for duodenal aspirate smears was employed for detection of coccidian protozoa oocysts namely Cryptosporidium parvum, Cyclospora cayetanensis and Cystoisospora belli.

Immunodiagnostic technique for detection of coproantigen, according to R-Biopharm AG, Darmstadt, Germany, which is certified by the Quality Control.

Fresh or frozen duodenal aspirate samples were subjected to rapid immunochromatographic test for detection of Cryptosporidium and/or Giardia antigens(Rida quick Cryptosporidium/Giardia Combi Art. No. N1122).

This test is a quick immunochromatographic test for the qualitative determination of Cryptosporidium parvum and/or Giardia lamblia in samples. Antigens from clinical specimens that are specific for these two parasites are isolated and immobilized on a membrane using specific antibodies. An antibody-enzyme conjugated then binded to specific sites on these antigens.

The antigens were detected after the addition of substrate by the formation of color bars in different areas depending on the parasite present and showed on the test device as red and blue bands. 


\section{Results and Discussion}

\section{Positive}

A) Cryptosporidium parvum positive: a blue test band appears along with the green control band.

B) Giardia lamblia positive: a red test band appears along with the green control band.

C) Cryptosporidium and Giardia positive: blue and red bands appear along with the green control band.

\section{Negative}

Only the green control band appears.

\section{Not valid}

No green control band. In this case, the test must be repeated with a new strip.

\section{Histopathological examination}

Collection of biopsy samples (Koch and Zurad, 2020)

Biopsy samples are taken when visible changes are seen in the duodenum during upper endoscopy.

Specimens were then subjected to microscopic examinations of the section stained by hematoxylin and eosin stain to detect inflammation, granuloma, eosinophilia and parasitic infections of the duodenum (Allen, 1992).

\section{Statistical analysis}

Statistical presentation and analysis of the present study was conducted, using chi-square test by SPSS V.22. For categorical variables, Chi-square test was used for analysis and when it was found inappropriate, it was replaced by Ficher Exact Test. $\mathrm{P}$ value was considered statistically significant when < 0.05

\section{Results and Discussion}

\section{Demographic data of cases with parasitic infections}

Parasitic infections were detected in only 15 patients $(21.4 \%)$. Two patients $(13.3 \%)$ were $<40$ years, nine patients $(60 \%)$ were from $(40-60)$ years and four patients $(26.7 \%)$ were $>60$ years.

Moreover, 11 patients $(73.3 \%)$ were males and four patients $(26.7 \%)$ were females. Concerning residence, 14 patients (93.3\%) were from rural areas and only one patient $(6.7 \%)$ was from urban area (Table 1).

\section{Parasites detected via direct observation by endoscopy}

Ascaris lumbricoides adult worm was directly observed in one case only $(1.4 \%)$ by the endoscope in the duodenum. The worm was extracted during endoscopy and was then identified by its gross macroscopic features (Figure 1).

Parasites detected by examination of duodenal aspiration and biopsy samples

By laboratory examination of duodenal aspirate and biopsy samples, the following parasites were detected (Table 2).

G. duodenalis was detected in 8 cases including one case was mixed $G$. duodenalis and Cryptosporidium spp. infection.

Cryptosporidium spp. was detected in 6 cases including one case mixed G. duodenalis and Cryptosporidium and another case mixed Cryptosporidium and Cyclospora cayetanensis infection. 
Performance of different diagnostic techniques

Regarding different methods of duodenal aspiration and biopsy examination used in the diagnosis of parasitic infection, only three cases $(4.3 \%)$ were positive by direct smear examination of duodenal aspirate and nine cases $(12.9 \%)$ were positive by using iodine staining. By modified Ziehl-Neelsen stain, six cases $(8.6 \%)$ were found positive.

As regards the immunoassay technique for antigen detection, seven strips (10\%) showed positive results, while by using histopathological examination of biopsies, 13 specimens (18.6\%) were found positive (Table 3).

\section{Direct smear examination}

Regarding direct smear examination, this study had detected three positive cases (4.3\%) for $G$. duodenalis with a statistically significant difference $(P<0.001)$. No positive cases were detected for Cryptosporidium spp., C. cayetanensis and $E$. histolytica infection by direct smear examination (Table 4).

\section{Iodine stain examination}

Using iodine stain, G. duodenalis cysts were detected in eight cases (11.4\%) (Figure2). In addition, E. histolytica metacyst was detected in one case $(1.4 \%)$. These results were statistically significant $(P<0.001$ in $G$. duodenalis and 0.009 in E. histolytica) (Table 5).

\section{Intestinal coccidian infections by modified Ziehl-Neelsen (Z.N.) stain}

Regarding modified Z.N. stain, Cryptosporidium oocysts were detected in six cases (Figure 3), while C. cayetanensis oocyst was detected in one case only (Figure 4) with a rate of $8.6 \%$ and $1.4 \%$, respectively. ZiehlNeelsen stain showed statistically significant results in the diagnosis of both Cryptosporidium and Cyclospora infection $(P<0.001)($ Table 6).

\section{Immunoassay technique for antigen detection}

By the use of the immunoassay technique, seven strips were found positive. Four strips (5.7\%) were positive for $G$. duodenalis only, two strips (2.9\%) were positive for Cryptosporidium spp. only and one strip was positive for both infections (1.4\%). The detection of antigen was found to be statistically significant for the diagnosis of $G$. duodenalis and Cryptosporidium spp. ( $P<$ 0.001 and $P<0.02$, respectively) (Table 7 , figure5).

\section{Histopathological examination of biopsy samples}

Among the 70 duodenal mucosal specimens, pear-shaped G. duodenalis trophozoites were detected in eight cases (11.4\%). These specimens also showed histopathological features of infection such as infiltration by chronic inflammatory cells and eosinophils as well as villous atrophy (Figures 6 and 7). In addition, goblet cell hyperplasia is found (Figure 6). Cryptosporidium intracellular stages were also found in six cases $(8.6 \%)$. These cases showed infiltration by chronic inflammatory cells and eosinophils, flattened fused villi (Figures 8 and 9) as well as goblet cell hyperplasia (Figure 8). Cyclospora cayetanensis and E. histolytica were both negative on histopathological examination. These results were statistically significant $(P<$ $0.001)$ for the diagnosis of $G$. duodenalis and Cryptosporidium spp. infection (Table 8). 
Table.1 Demographic data of cases with parasitic infections

\begin{tabular}{|c|c|c|c|}
\hline \multicolumn{3}{|c|}{ Demographic data } & \multirow{2}{*}{$\begin{array}{c}\text { Positive cases } \\
2\end{array}$} \\
\hline \multirow[t]{6}{*}{ Age } & \multirow[t]{2}{*}{$<40$} & $\mathbf{N}$ & \\
\hline & & $\%$ & $13.3 \%$ \\
\hline & \multirow[t]{2}{*}{$40-60$} & $\mathbf{N}$ & 9 \\
\hline & & $\%$ & $60 \%$ \\
\hline & \multirow[t]{2}{*}{$>60$} & $\mathbf{N}$ & 4 \\
\hline & & $\%$ & $26.7 \%$ \\
\hline \multirow[t]{4}{*}{ Gender } & \multirow[t]{2}{*}{ Male } & $\mathbf{N}$ & 11 \\
\hline & & $\%$ & $73.3 \%$ \\
\hline & \multirow[t]{2}{*}{ Female } & $\mathbf{N}$ & 4 \\
\hline & & $\%$ & $26.7 \%$ \\
\hline \multirow[t]{3}{*}{ Residence } & \multirow[t]{2}{*}{ Rural } & $\mathbf{N}$ & 14 \\
\hline & & $\%$ & $93.3 \%$ \\
\hline & Urban & $\mathbf{N}$ & 1 \\
\hline
\end{tabular}

Table.2 Parasites detected by examination of duodenal aspiration and biopsy samples

\begin{tabular}{|c|c|}
\hline Parasites detected & No. of cases \\
\hline Giardia duodenalis & 8 \\
\hline Cryptosporidium spp. & 6 \\
\hline Cyclospora cayetanensis & 1 \\
\hline Entamoeba histolytica & 1 \\
\hline
\end{tabular}

Table.3 Performance of different diagnostic techniques

\begin{tabular}{|c|c|c|c|c|c|c|}
\hline \multicolumn{2}{|c|}{ Methods } & Direct smear & Iodine & Z.N. & Immunoassay & Biopsy \\
\hline \multirow[t]{2}{*}{ Positive } & $\mathbf{N}$ & 3 & 9 & 6 & 7 & 13 \\
\hline & $\%$ & $4.3 \%$ & $12.9 \%$ & $8.6 \%$ & $10 \%$ & $18.6 \%$ \\
\hline \multirow[t]{2}{*}{ Negative } & $\mathbf{N}$ & 67 & 61 & 64 & 63 & 57 \\
\hline & $\%$ & $95.7 \%$ & $87.1 \%$ & $91.4 \%$ & $90 \%$ & $81.4 \%$ \\
\hline \multirow[t]{2}{*}{ Total } & $\mathbf{N}$ & 70 & 70 & 70 & 70 & 70 \\
\hline & $\%$ & $100.0 \%$ & $100.0 \%$ & $100.0 \%$ & $100.0 \%$ & $100.0 \%$ \\
\hline
\end{tabular}

Table.4 Findings of direct smear examination

\begin{tabular}{|c|c|c|c|c|c|c|}
\hline & & & \multicolumn{2}{|c|}{ Direct smear } & \multirow[t]{2}{*}{$\mathbf{X}^{2}$} & \multirow[t]{2}{*}{$P$ value } \\
\hline & & & +ve & -ve & & \\
\hline \multirow[t]{4}{*}{ G. duodenalis } & \multirow[t]{2}{*}{+ ve } & $\mathbf{N}$ & 3 & 5 & \multirow[t]{4}{*}{24.290} & \multirow[t]{4}{*}{$0.001 *$} \\
\hline & & $\%$ & $4.3 \%$ & $7.1 \%$ & & \\
\hline & \multirow[t]{2}{*}{-ve } & $\mathbf{N}$ & 0 & 62 & & \\
\hline & & $\%$ & $.0 \%$ & $88.6 \%$ & & \\
\hline \multirow[t]{4}{*}{ Cryptosporidium spp. } & \multirow[t]{2}{*}{$+v e$} & $\mathbf{N}$ & 0 & 6 & \multirow[t]{4}{*}{0.294} & \multirow[t]{4}{*}{0.588} \\
\hline & & $\%$ & $.0 \%$ & $8.6 \%$ & & \\
\hline & \multirow[t]{2}{*}{-ve } & $\mathbf{N}$ & 3 & 61 & & \\
\hline & & $\%$ & $4.3 \%$ & $87.1 \%$ & & \\
\hline \multirow[t]{4}{*}{ C. cayetanensis } & \multirow[t]{2}{*}{$+v e$} & $\mathbf{N}$ & 0 & 1 & \multirow[t]{4}{*}{0.045} & \multirow[t]{4}{*}{0.831} \\
\hline & & $\%$ & $.0 \%$ & $1.4 \%$ & & \\
\hline & \multirow[t]{2}{*}{-ve } & $\mathbf{N}$ & 3 & 66 & & \\
\hline & & $\%$ & $4.3 \%$ & $94.3 \%$ & & \\
\hline \multirow[t]{4}{*}{ E. histolytica } & \multirow[t]{2}{*}{$+v e$} & $\mathbf{N}$ & 0 & 1 & \multirow[t]{4}{*}{0.045} & \multirow[t]{4}{*}{0.831} \\
\hline & & $\%$ & $.0 \%$ & $1.4 \%$ & & \\
\hline & \multirow[t]{2}{*}{-ve } & $\mathbf{N}$ & 3 & 66 & & \\
\hline & & $\%$ & $4.3 \%$ & $94.3 \%$ & & \\
\hline
\end{tabular}

* Significant $(P$ value $<0.05)$ 
Table.5 Findings of iodine stain examination

\begin{tabular}{|c|c|c|c|c|c|c|}
\hline & & & \multicolumn{2}{|c|}{ Iodine } & \multirow[t]{2}{*}{$\mathbf{X}^{2}$} & \multirow[t]{2}{*}{$P$ valuc } \\
\hline & & & +ve & -ve & & \\
\hline \multirow[t]{4}{*}{ G. duodenalis } & \multirow[t]{2}{*}{+ ve } & $\mathbf{N}$ & 8 & 0 & \multirow[t]{4}{*}{61.219} & \multirow[t]{4}{*}{$0.001 *$} \\
\hline & & $\%$ & $11.4 \%$ & $.0 \%$ & & \\
\hline & \multirow[t]{2}{*}{-ve } & $\mathbf{N}$ & 1 & 61 & & \\
\hline & & $\%$ & $1.4 \%$ & $87.1 \%$ & & \\
\hline \multirow[t]{4}{*}{ E. histolytica } & \multirow[t]{2}{*}{+ ve } & $\mathbf{N}$ & 1 & 0 & \multirow[t]{4}{*}{6.879} & \multirow[t]{4}{*}{$0.009 *$} \\
\hline & & $\%$ & $1.4 \%$ & $.0 \%$ & & \\
\hline & \multirow[t]{2}{*}{-ve } & $\mathbf{N}$ & 8 & 61 & & \\
\hline & & $\%$ & $11.4 \%$ & $87.1 \%$ & & \\
\hline
\end{tabular}

* Significant $(P$ value $<0.05)$

Table.6 Intestinal coccidian infections by modified Z.N. stain

\begin{tabular}{|c|c|c|c|c|c|c|}
\hline & & & \multicolumn{2}{|c|}{ Z.N. } & \multirow[t]{2}{*}{$X^{2}$} & \multirow[t]{2}{*}{$P$ valu } \\
\hline & & & $+\mathrm{ve}$ & -ve & & \\
\hline \multirow[t]{4}{*}{ Cryptosporidium spp. } & \multirow[t]{2}{*}{$+v e$} & $\mathbf{N}$ & 6 & 0 & \multirow[t]{4}{*}{70.001} & \multirow[t]{4}{*}{$0.001 *$} \\
\hline & & $\%$ & $8.6 \%$ & $.0 \%$ & & \\
\hline & \multirow[t]{2}{*}{-ve } & $\mathbf{N}$ & 0 & 64 & & \\
\hline & & $\%$ & $.0 \%$ & $91.4 \%$ & & \\
\hline \multirow[t]{4}{*}{ C. cayetanensis } & \multirow[t]{2}{*}{+ ve } & $\mathbf{N}$ & 1 & 0 & \multirow[t]{4}{*}{10.821} & \multirow[t]{4}{*}{$0.001 *$} \\
\hline & & $\%$ & $1.4 \%$ & $.0 \%$ & & \\
\hline & \multirow[t]{2}{*}{-ve } & $\mathbf{N}$ & 5 & 64 & & \\
\hline & & $\%$ & $7.1 \%$ & $91.4 \%$ & & \\
\hline
\end{tabular}

Table.7 Immunoassay technique for antigen detection

\begin{tabular}{|c|c|c|c|c|c|c|}
\hline & & & \multicolumn{2}{|c|}{ Immunoassay } & \multirow[t]{2}{*}{$X^{2}$} & \multirow[t]{2}{*}{$P$ value } \\
\hline & & & $+\mathrm{ve}$ & -ve & & \\
\hline \multirow[t]{4}{*}{ G. duodenalis } & \multirow[t]{2}{*}{$+\mathrm{ve}$} & $\mathbf{N}$ & 4 & 3 & \multirow[t]{4}{*}{19.213} & \multirow[t]{4}{*}{$0.001 *$} \\
\hline & & $\%$ & $5.7 \%$ & $4.3 \%$ & & \\
\hline & \multirow[t]{2}{*}{-ve } & $\mathbf{N}$ & 3 & 60 & & \\
\hline & & $\%$ & $4.3 \%$ & $85.7 \%$ & & \\
\hline \multirow[t]{4}{*}{ Cryptosporidium spp. } & \multirow[t]{2}{*}{+ ve } & $\mathbf{N}$ & 2 & 3 & \multirow[t]{4}{*}{5.379} & \multirow[t]{4}{*}{$0.020 *$} \\
\hline & & $\%$ & $2.9 \%$ & $4.3 \%$ & & \\
\hline & \multirow[t]{2}{*}{-ve } & $\mathbf{N}$ & 5 & 60 & & \\
\hline & & $\%$ & $7.1 \%$ & $85.7 \%$ & & \\
\hline \multirow{4}{*}{$\begin{array}{c}\text { G. duodenalis \& } \\
\text { Cryptosporidium spp. }\end{array}$} & \multirow[t]{2}{*}{+ ve } & $\mathbf{N}$ & 1 & 3 & \multirow[t]{4}{*}{1.058} & \multirow[t]{4}{*}{0.303} \\
\hline & & $\%$ & $1.4 \%$ & $4.3 \%$ & & \\
\hline & \multirow[t]{2}{*}{-ve } & $\mathbf{N}$ & 6 & 60 & & \\
\hline & & $\%$ & $8.6 \%$ & $85.7 \%$ & & \\
\hline
\end{tabular}


Table. 8 Histopathological examination of biopsy samples

\begin{tabular}{|c|c|c|c|c|c|c|}
\hline & & & \multicolumn{2}{|c|}{ Biopsy } & \multirow[t]{2}{*}{$X^{2}$} & \multirow[t]{2}{*}{$P$ value } \\
\hline & & & + ve & -ve & & \\
\hline \multirow[t]{4}{*}{ G. duodenalis } & \multirow[t]{2}{*}{$+v e$} & $\mathbf{N}$ & 8 & 0 & \multirow[t]{4}{*}{39.603} & \multirow[t]{4}{*}{$0.001 *$} \\
\hline & & $\%$ & $11.4 \%$ & $.0 \%$ & & \\
\hline & \multirow[t]{2}{*}{-ve } & $\mathbf{N}$ & 5 & 57 & & \\
\hline & & $\%$ & $7.1 \%$ & $81.4 \%$ & & \\
\hline \multirow[t]{4}{*}{ Cryptosporidium spp. } & \multirow[t]{2}{*}{$+v e$} & $\mathbf{N}$ & 6 & 0 & \multirow[t]{4}{*}{28.774} & \multirow[t]{4}{*}{$0.001 *$} \\
\hline & & $\%$ & $8.6 \%$ & $.0 \%$ & & \\
\hline & \multirow[t]{2}{*}{-ve } & $\mathbf{N}$ & 7 & 57 & & \\
\hline & & $\%$ & $10.0 \%$ & $81.4 \%$ & & \\
\hline \multirow[t]{4}{*}{ C. cayetanensis } & \multirow[t]{2}{*}{$+\mathrm{ve}$} & $\mathbf{N}$ & 0 & 1 & \multirow[t]{4}{*}{0.231} & \multirow[t]{4}{*}{0.631} \\
\hline & & $\%$ & $.0 \%$ & $1.4 \%$ & & \\
\hline & \multirow[t]{2}{*}{-ve } & $\mathbf{N}$ & 13 & 56 & & \\
\hline & & $\%$ & $18.6 \%$ & $80.0 \%$ & & \\
\hline \multirow[t]{4}{*}{ E. histolytica } & \multirow[t]{2}{*}{$+\mathrm{ve}$} & $\mathbf{N}$ & 0 & 1 & \multirow[t]{4}{*}{0.231} & \multirow[t]{4}{*}{0.631} \\
\hline & & $\%$ & $.0 \%$ & $1.4 \%$ & & \\
\hline & \multirow[t]{2}{*}{-ve } & $\mathbf{N}$ & 13 & 56 & & \\
\hline & & $\%$ & $18.6 \%$ & $80.0 \%$ & & \\
\hline
\end{tabular}

Table.9 Association between symptomatology and parasite-positive cases

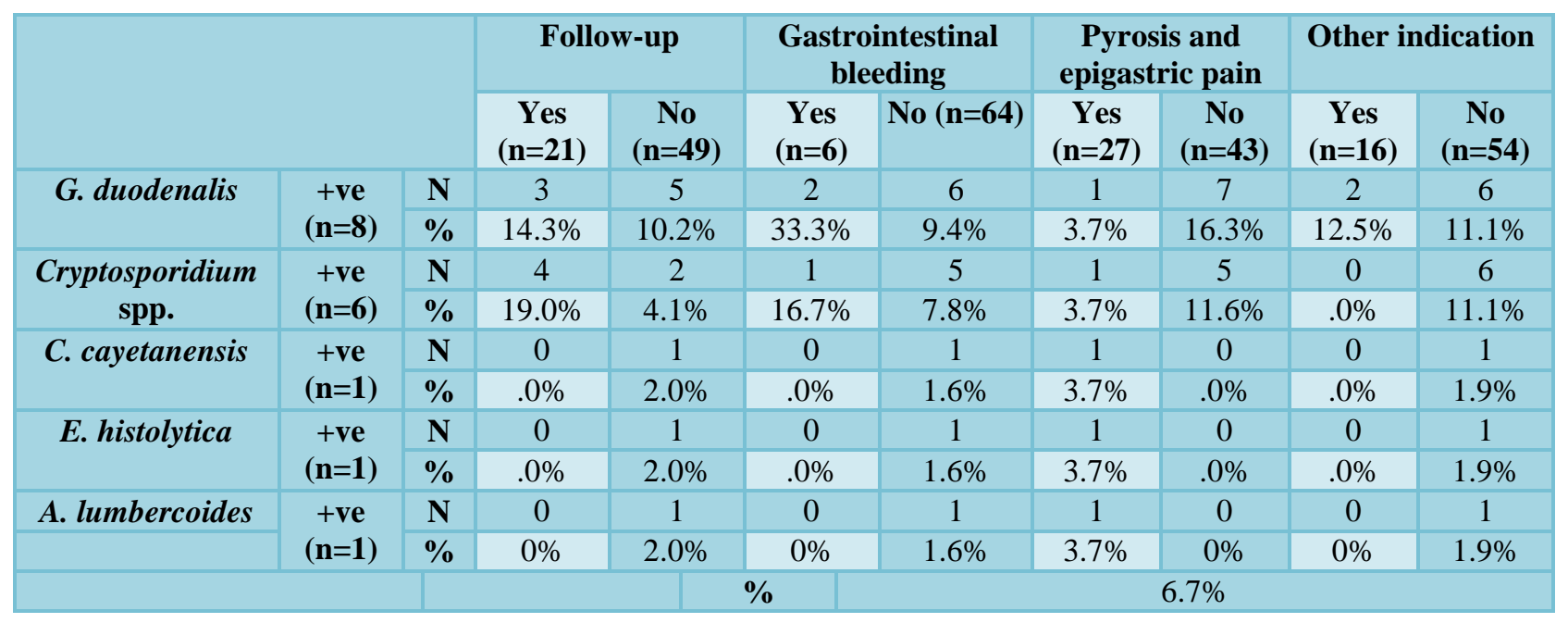

Figure.1 Ascaris lumbricoides detected directly by endoscopy

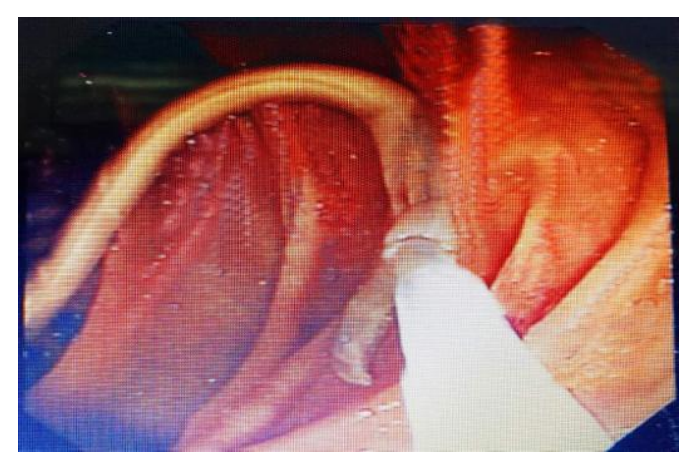


Figure.2 Iodine-stained duodenal aspirate sample showing G. duodenalis cysts (arrows) (× 1000)

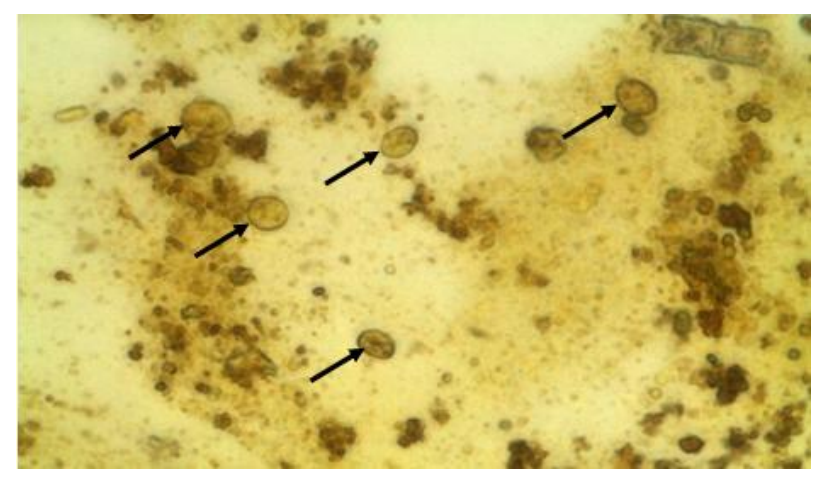

Figure.3 Duodenal aspirate sample showing Cryptosporidium oocysts (arrows) with modified Z.N. stain $(\times 1000)$

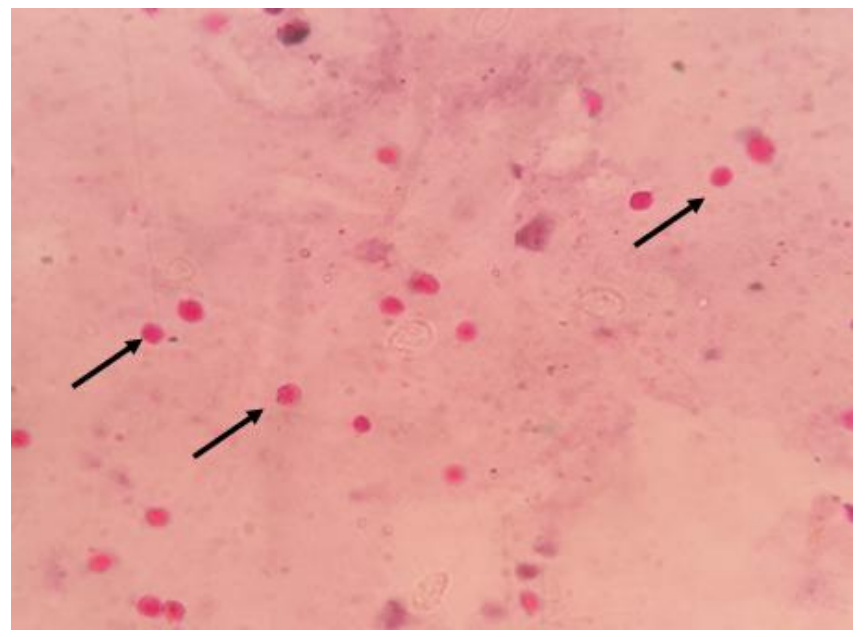

Figure.4 Duodenal aspirate sample showing $C$. cayetanensis oocysts (arrows) by modified Z.N. stain $(\times 1000)$

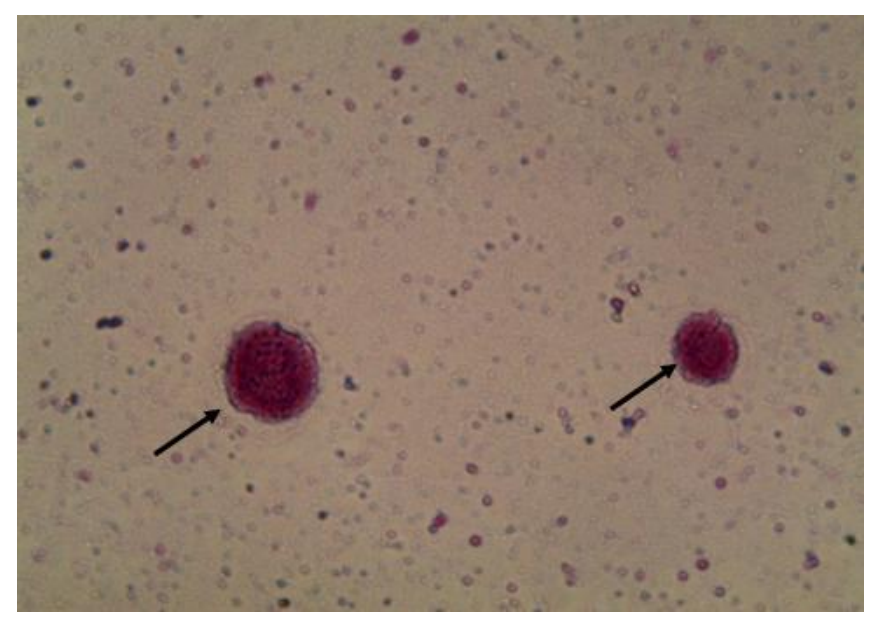


Figure.5 Immunochromatographic strips (Cryptosporidium/ Giardia Combi), (1) positive for both $C$. parvum and $G$. duodenalis, (2) positive for $C$. parvum and (3) positive for $G$. duodenalis

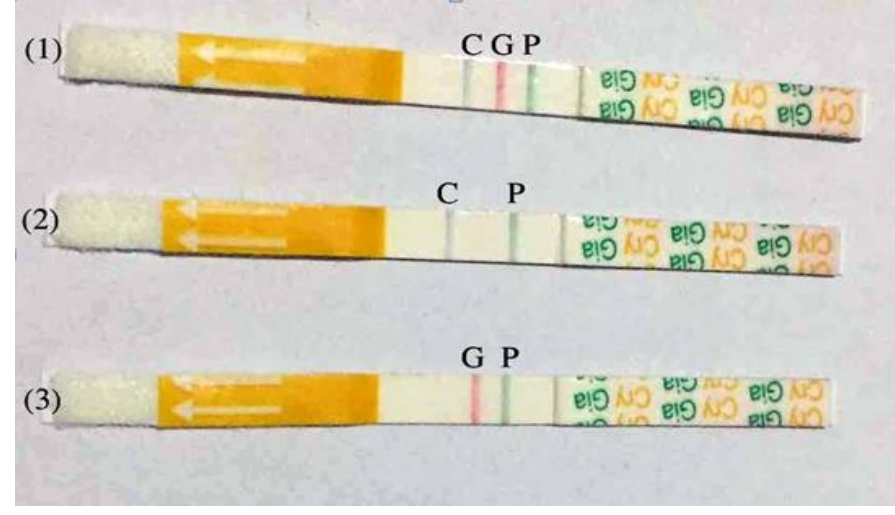

(C): Cryptosporidium parvum; (G): Giardia duodenalis; (P): Positive control

Figure.6 Micrograph of a duodenal mucosal biopsy illustrating the presence of pear-shaped $G$. duodenalis trophozoites overlying the epithelium (black arrows), chronic inflammatory cells in the core of flattened villi (yellow arrow) as well as goblet cell hyperplasia (red arrows)

$(\mathrm{H} \& \mathrm{E} \times 400)$

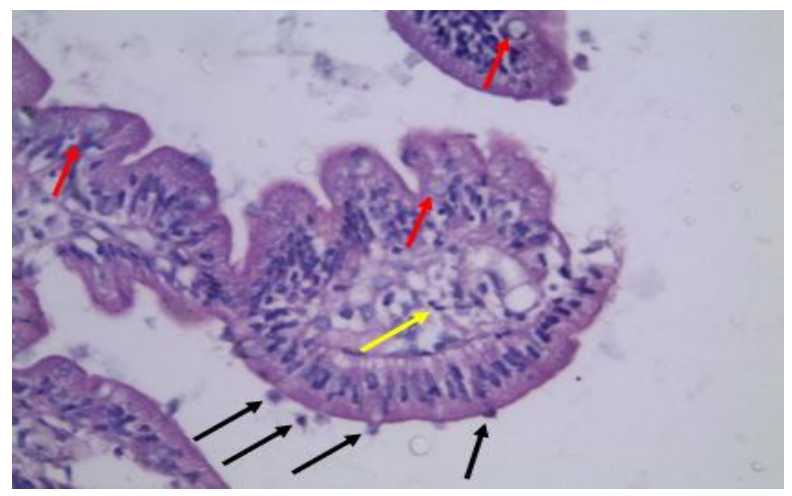

Figure.7 Micrograph of a duodenal mucosal biopsy showing G. duodenalis trophozoites (black arrows) as well as chronic inflammatory cells and eosinophils (yellow arrow) in the core of villi $(\mathrm{H} \& \mathrm{E} \times 400)$

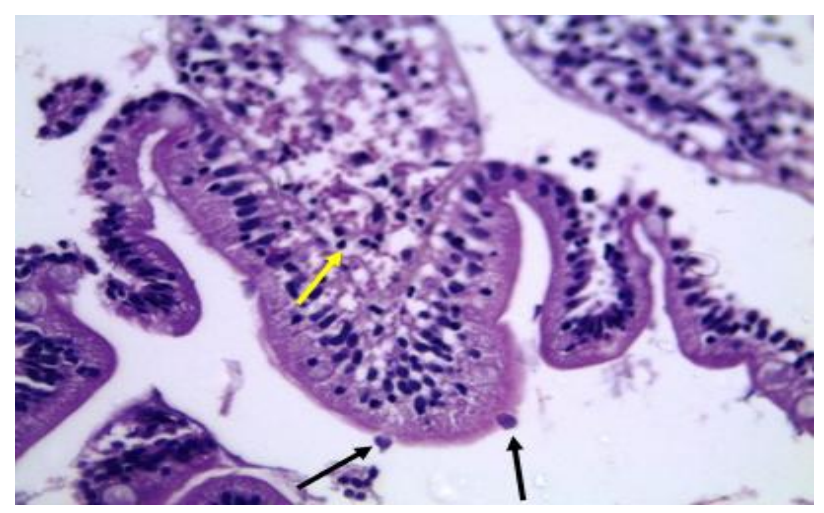


Figure.8 Micrograph of a duodenal mucosal biopsy in Cryptosporidium-positive case showing fused flattened villi (black arrow) as well as goblet cell hyperplasia (red arrow) $(\mathrm{H} \& \mathrm{E} \times 100)$

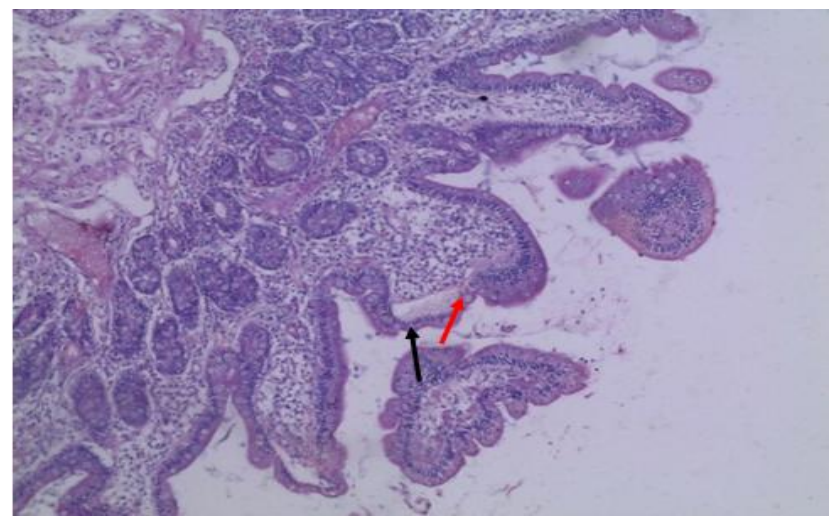

Figure.9 Micrograph of a duodenal mucosal biopsy in Cryptosporidium-positive case showing infiltration by chronic inflammatory cells and eosinophils (arrow) $(\mathrm{H} \& \mathrm{E} \times 400)$

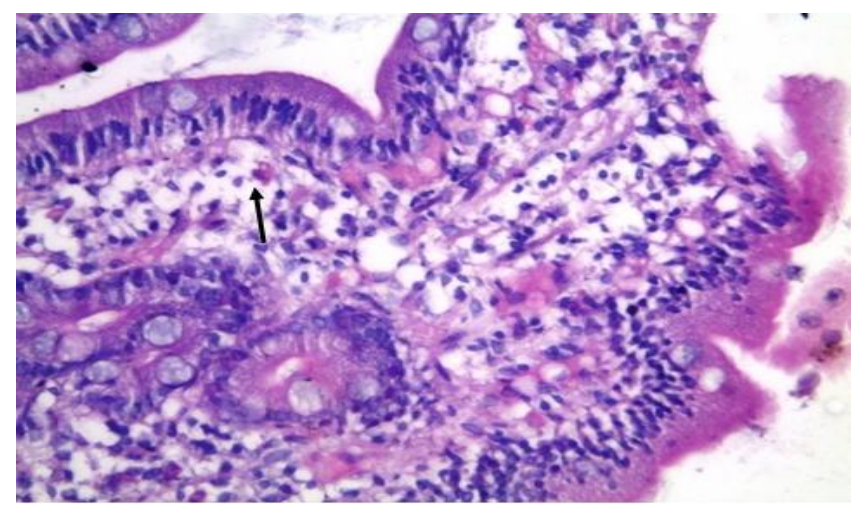

Parasitic diseases caused by helminths and protozoan parasites constitute significant but neglected public health problems in the developing countries and marginalized communities (Devleesschauwer et al., 2014). Diagnosis of gastrointestinal parasitic infections is commonly based on coprology for detection of eggs or other parasitic stages in the stools and/or elevated count of eosinophils in blood picture (Mohamed et al., 2000). Thus, false negative cases are possible when the aforementioned findings are missed. Serological assays also are available for the diagnosis of parasitic infections but cross reactivity limits their sensitivity and specificity. Therefore, histopathological examination may be a reliable way to diagnose parasitic diseases by detecting the characteristic morphology of parasites. However, failure to demonstrate the parasite does not exclude the possibility of infection where inflammatory reaction in the surrounding tissue could give a clue about parasitic infestations (Mohamed et al., 1997; Rosenblatt et al., 2009).

In the current study, there is a considerable range of reported prevalence of parasitic infections in duodenal aspiration and biopsy by upper gastrointestinal endoscopy, which likely reflects differences in demographic factors, parasite endemicity, and differences in the performance of diagnostic techniques used. 
As regards our study, the ratio of parasitic infections was higher $(60 \%)$ in the age group ranging from (40-60) years. This finding is consistent with a study involving clinical, endoscopic and histopathological profile of parasitic duodenitis done by Santos et al., (2011) who found that the mean age for detected Strongyloides stercoralis and Cryptosporidium-positive cases was 32.4 and 43.8 years, respectively.

Concerning gender in this study, the incidence of parasitic infections was generally higher in males $(73.3 \%)$ than females $(26.7 \%)$ and this coincides with a retrospective study on detection of parasitic infections during upper gastrointestinal endoscopic procedures by Zaher et al., (2012), where $62.5 \%$ of detected cases were males and $37.5 \%$ were females. Similarly, Santos et al., (2011) reported that $54.5 \%$ of detected cases were males. This may be related to the higher risk of exposure in males than females.

This study was conducted in Gharbia Governorate, which is considered a ruralurban area (El-Khoby et al., 2000). Unsurprisingly, most of detected parasites in this work are found in rural areas, where 93.3\% of positive cases were of rural origin. This coincides with Zaher et al., (2012), where all cases of parasitic infections were of rural origin. Likewise, many studies had reported higher incidence of parasitic infections in rural communities in comparison to urban communities (Ikeh et al., 2006; Ngrenngarmlert et al., 2007). This may be explained by lack of health education, lack of proper sewage disposal systems, poor hygiene as well as exposure to soil and farm animals.

In the current research, parasitic infections were reported in 15 patients out of 70 studied cases $(21.4 \%)$. One patient was diagnosed by direct observation of Ascaris lumbricoides by endoscopy and 14 patients were diagnosed by examination of duodenal aspiration and biopsy samples. Thus, upper gastrointestinal endoscopy may have an important role in the diagnosis of parasitic infections.

Along the same line of evidence, a study by Carswell et al., (1973) on giardiasis and celiac disease, found that $50 \%$ of Giardia-positive cases were missed by stool examination alone. Moreover, Ament et al., (1973) had studied the structure and function of gastrointestinal tract in primary immunodeficiency syndromes in 39 patients by examination of stools and small intestinal biopsies for Giardia cyst or trophozoite. They mentioned that only $30 \%$ of their cases of giardiasis were detected by stool examination alone, the remainder being identified by small bowel biopsy. Mahdi and Taha, (2002) revealed the presence of $G$. lamblia trophozoites in duodenal aspirates taken from 15 patients, five of whom had positive stool examination. Also, C. parvum was recovered from four patients, compared to only two cases discovered by stool examination. They mentioned that rapid examination of duodenal aspirate besides its clear liquid nature considerably facilitates the recognition of trophozoites.

Moreover, Roberts et al., (1989) reported an unusually high prevalence of cryptosporidiosis (12.7\%) in duodenal aspirates from immunocompetent patients during routine upper endoscopy, while stool samples were positive in less than $50 \%$ of those adults with positive duodenal aspirates. In addition, in a study by Mohamed et al., (2000), gastroscopy showed multiple Ascaris lumbricoides worms in one patient presented with abdominal pain and vomiting.

However, Nair et al., (1977) and Grazioli et al., (2006) showed that stool examination is as accurate as the direct smear examination of the duodenal samples in diagnosing $G$. 
lamblia. On the contrary to the high percentage of parasitic infections in our study, Zaher et al., (2012) reported lower incidence where only $0.16 \%$ of their participants were incidentally diagnosed with parasitic infection during upper endoscopic examination for other reasons. This may be explained by different method of diagnosis where all cases were diagnosed by macroscopic detection of adult worm by endoscopy and not by microscopic examination of duodenal aspirate or biopsy samples. Furthermore, Hanevik et al., (2007), on studying persisting symptoms and duodenal inflammation related to $G$. duodenalis infection, found Giardia trophozoites in duodenal biopsies in only 4 (10\%) of the 40 cases with Giardia-positive faecal samples while duodenal aspirate microscopy showed no positive results. The explanation for the poor outcome may be due to the procedure of duodenal aspiration where $\mathrm{NaCl}$-instillation then suction of fluid rather than direct suction or may be due to long standing or subsiding infection and previous treatment.

Regarding direct smear examination, our study had detected three positive cases for G.duodenalis with a rate of $4.3 \%$, while no positive cases for Cryptosporidium spp., $C$. cayetanensis or E. histolytica were detected by direct smear examination. Sensitivity of direct smear in diagnosis of $G$. duodenalis was found to be $37.5 \%$, whereas specificity for G.duodenalis, Cryptosporidium spp., $C$. cayetanensis and E. histolytica was found to be $100 \%, 95.3 \%, 95.7 \%$ and $95.7 \%$, respectively.

Upon considering results of iodine and modified Z.N. staining of duodenal aspirate as the gold standard tests, iodine staining was highly sensitive in the diagnosis of $G$. duodenalis and E. histolytica (100\% for both) and highly specific $(98.4 \%$ and $88.4 \%$, respectively). Modified Z.N. staining was highly sensitive for diagnosing Cryptosporidium spp. and $C$. cayetanensis (100\% for both parasites) and highly specific (100\% and $92.8 \%$, respectively). In a similar study by Kerlin et al., (1978) for the detection of Giardia by upper endoscopy showed that the prevalence of Giardia was (2.1\%) in both duodenal aspirate and mucosal immersion smears. Furthermore, El-Hady et al., (2018) studied the parasites in duodenal aspirate using direct wet and modified acid-fast stained smears in Sohag, Egypt. Cryptosporidium and Giardia cases were detected in duodenal aspirate samples with ratio $44 \%$ and $8 \%$, respectively. Cyclospora cases were detected in $11 \%$ of duodenal aspirate samples. In addition, Blastocystis hominis was detected in $21 \%$ of patients.

However, Chakarova (2010) detected $G$. duodenalis and Cryptosporidium in human stool and duodenal aspirate by direct smear examination where sensitivity was $97.9 \%$ and $86.5 \%$, respectively. In addition, Elsafi et al., (2013) reported that microscopic examination of iodine-stained stool samples and Kinyoun's acid-fast technique for detection of G. lamblia and $C$. parvum, respectively were less sensitive and less specific than ImmunoCard STAT® and real-time PCR analysis.

Concerning the use ofrapid immunodiagnostic techniques (RDT), they are rapid to perform and do not require experienced staff or special technical equipment and could be adopted as a screening test especially for smaller and less well-equipped labs (Regnath et al., 2006). The present study had detected seven cases by immunoassay technique (Rida quick Cryptosporidium/Giardia Combi): four cases (5.7\%) positive for G. duodenalis, two cases (2.9\%) positive for Cryptosporidium spp., and one case $(1.4 \%)$ positive for both infections. Sensitivity of antigen detection in the diagnosis of $G$. duodenalis and Cryptosporidium spp. is moderate $(62.5 \%$ and 
$50 \%$, respectively) and specificity is high (96.8\% and $93.8 \%$, respectively), but still not valid test for diagnosis as sensitivity $<80 \%$ ).

Johnston et al., (2003) showed comparable results for Giardia and Cryptosporidium using the ImmunoCard STAT rapid assay for the detection of Giardia and Cryptosporidium in stool with sensitivity $(81.3 \%$ and $67.6 \%)$ and specificity $(99.5 \%$ and $99.0 \%)$, respectively. On the contrary, Goudal et al., (2019) showed that copro-antigen detection in giardiasis and cryptosporidiosis using RDT was $89.2 \%$ sensitive and $99.3 \%$ specific for the diagnosis of $G$. intestinalis as it detected 33 out of the 37 cases detected by the microscopic examination of stool. While for Cryptosporidium spp., it was $86.7 \%$ sensitive and $100 \%$ specific as it detected 26 out of the 30 samples detected by the microscopic examination of stool. Similarly, Regnath et al., (2006) found that all microscopically positive specimens were also positive by both the RidaQuick Giardia and RidaQuick Crypto/Giardia Combi assay. Thus, the sensitivity values of the RidaQuick single species and Combi assays were 100\% for both parasites compared with microscopy. The specificity was $99 \%$ for G. lamblia in the single species and Combi assays, while it was $100 \%$ for Cryptosporidia in both assays. These differences can be explained by the low burden of parasites in our samples.

This study included histopathological examination of duodenal mucosal specimens from all 70 patients. G. duodenalis trophozoites were detected in eight cases (11.4\%) and Cryptosporidium spp. intracellular stages were also detected in six cases $(8.6 \%)$. These specimens also showed histopathological features of parasitic infections as infiltration by chronic inflammatory cells and eosinophils as well as villous atrophy.
Various studies were done to correlate histopathological changes in parasitic infections via endoscopic biopsies and had showed variable results. Similar to our findings, Mohamed et al., (2000) studied the histological diagnosis of parasitic infections from endoscopic biopsies and surgical specimens. G. lamblia was isolated from duodenal biopsies in eight patients. Villous atrophy was detected in a patient with malabsorption. Other findings were nonspecific and showed only inflammation, erosions, or whitish nodules. In four of those patients, repeated stool examinations did not show G. lamblia. They mentioned that the diagnosis of gastrointestinal parasites is not only made by stool examination but can be detected by histopathological examination of endoscopic biopsies or surgical specimens. Moreover, Hanevik et al., (2007) reported that duodenal biopsies in 57 patients $(47.1 \%)$ showed inflammation with oedema and infiltration of leukocytes, increased number of plasma cells in the lamina propria as well as architectural distortion with shortening and blunting of intestinal villi. Giardia trophozoites were visible in duodenal biopsies in only $4(10 \%)$ of the 40 cases with Giardiapositive faecal samples.

Furthermore, in a retrospective analysis of parasitic diseases diagnosed by tissue biopsy, giardiasis was seen only in three cases $(2 \%)$. Pear-shaped trophozoites were found in duodenal mucosa. All cases showed normal villous to crypt ratio with only increase in intraepithelial lymphocytes. They revealed that giardiasis is associated with various histological changes, which range from minimal to severe enterocyte damage, villous atrophy and crypt hyperplasia (Manandhar et al., 2018).

However, Santos et al., (2011) detected parasites in only $1 \%$ of duodenal biopsies performed and concluded that no endoscopic 
or histopathological features were characteristic to parasitic duodenitis. Moreover, Doganci et al., (2002) found only one positive duodenal aspirate sample for $C$. parvum while duodenal biopsies were negative in all samples. This wide range of variable results may be explained by different virulence of detected strains, host factors, or differences in the duration and severity of infection. On the contrary to our results, Zafar et al., (1991) investigated the frequency of $G$. lamblia in patients admitted for upper endoscopy for various causes and revealed that G. lamblia has been obtained in $9 \%$ of duodenal aspirates and in only $1.8 \%$ of biopsy specimens. They mentioned three factors which may be responsible for the low yield. Firstly, the size of the endoscopic biopsy, secondly, the staining technique and lastly, the third factor is the excessive use of antiprotozoal drugs and antibiotics in their country.

Furthermore, Grazioli et al., (2006) reported that histological examination of duodenal biopsies for G.lamblia was unsuitable due to an unacceptable rate of false negative results (e.g., 22.2\% sensitivity). Manandhar et al., (2018) detected amoebiasis in $2 \%$ of total positive cases where histopathology showed round trophozoite with ingested erythrocytes in the cytoplasm. However, E. histolytica trophozoites were not detected in our biopsies, which may be explained by different biopsy site where in our study biopsy was taken from duodenum while in their study biopsies were taken from colon.

This study included 70 patients admitted for upper gastrointestinal endoscopy unit for various indications. $G$. duodenalis and Cryptosporidium spp. were detected in patients admitted for haematemsis, pyrosis or epigastric pain, persistent vomiting or dysphagia and follow-up of chronic liver diseases, liver cirrhosis or esophageal varices.
Cyclospora cayetanensis and E. histolytica were detected in patients with pyrosis or epigastric pain. Ascaris lumbricoides adult was detected in patient manifested by recurrent epigastric pain.

Also, Zafar et al., (1991) found that $33.3 \%$ of patient with $G$. lamblia in duodenal aspirate were follow-up cases of duodenal ulcer. Jahani et al., (2008) studied a group of 130 dyspeptic patients who had undergone upper gastrointestinal endoscopy and Giardia was detected in two (1.5\%) patients. Moreover, Doganci et al., (2002) reported that dyspepsia was the main indication of upper endoscopy in the Cryptosporidium-positive case found in their study.

Furthermore, McHenry et al., (1987) on studying the yield of routine duodenal aspiration for $G$. lamblia during esophagogastroduodenoscopy, reported that the main indications for routine upper gastrointestinal endoscopy were abdominal pain53\%, gastroesophageal reflux symptoms $8 \%$, nausea/vomiting $6 \%$, gastrointestinal bleeding $6 \%$, diarrhea $6 \%$, ulcer follow-up $4 \%$, weight loss $3 \%$ and miscellaneous causes $14 \%$.

Similarly, Zaher et al., (2012) detected one case of A. lumbricoides in a patient manifested by epigastric pain. However, they correlated some other parasites diagnosed by upper endoscopy with other clinical presentations where Ancylostoma duodenale parasite was detected in five cases. Strongyloides stercoralis and Fasciola hepatica each was detected in one separate case.

All cases of Ancylostoma had anemia, four cases had hemoglobin level $<10 \mathrm{gm} \%$, Strongyloides case had mild anemia and epigastric pain while Fasciola presented with obstructive jaundice. 
In conclusion, our study highlighted the value of duodenal aspirate and mucosal biopsies examination for detection of intestinal parasites. Microscopic examination of the samples either by direct wet mount or by stained preparations, showed relatively accepted results. Antigen detection was found to be easy but still need further evaluation in more cases. Histopathological examination proved helpful in the diagnosis as it allowed the detection of both the parasite and its pathology.

\section{Recommendations}

It is recommended to collect and examine duodenal contents for parasitic infections as routine procedures during upper gastrointestinal endoscopy. Further studies should be done on larger number of patients and comparative studies between duodenal aspirate and stool examination for search of parasites are recommended. Elaboration of effective and practical techniques for evaluation of the impact of intestinal parasitic infections upon the pathology of the gastrointestinal tract is recommended.

\section{References}

Ali, I.K., Clark, C.G., Petri, W.A. (2008): Molecular epidemiology of amebiasis. Infect Genet Evol; 8(5): 698-707.

Allen, T. (1992): Hematoxylin and eosin. Laboratory methods in histotechnology. AFIP, Washington, DC; 53(1): 53-58.

Ament, M.E., Ochs, H.D., Davis, S.D. (1973): Structure and function of the gastrointestinal tract in primary immunodeficiency syndromes. A study of 39 patients. Medicine; 52(3): 227-248.

Bhaijee, F., Subramony, C., Tang, S. J., Pepper, D. J. (2011): Human immunodeficiency virus-associated gastrointestinal disease: common endoscopic biopsy diagnosis. Patholog Res Int; 2011:247923, 1-8.

Carleton, H.M., Dury, R.A., Wallington, E.A. (1980): Carleton's histological techniques, $5^{\text {th }}$ edition. Carleton, H.M., Dury, R.A., Wallington, E.A. (editors). Oxford University Press, New York, Vol. 243. pp. 417.

Carswell, F., Gibson, A.A., McAllister, T.A. (1973): Giardiasis and coeliac disease. Arch Dis Child; 48(6): 414-418.

Chakarova, B. (2010): Comparative evaluation of the diagnostic methods for detection of Giardia intestinalis in human fecal samples. Trakia J Sci; 8(2): 174-179.

Devleesschauwer, B., Ale, A., Torgerson, P., Praet, N., Maertens de Noordhout, C, et al., (2014): The burden of parasitic zoonoses in Nepal: a systematic review. PLoS Negl Trop Dis; 8(1): e2634.

Doganci, T., Araz, E., Ensari, A., Tanyuksel, M., Doganci, L. (2002): Detection of Cryptosporidium parvum infection in childhood using various techniques. Med Sci Monit; 8(12): 223-226.

El-Hady, H.A., Abd-Elmaged, S., AbdElmawgood, A.A., Ahmed, S.G. (2018): Parasites in duodenal aspirate using endoscopy in Sohag, Egypt. Sohag Med $\mathrm{J} ; 22(3): 239-244$.

El-Khoby, T., Galal, N., Fenwick, A., Barakat, R., El-Hawey, A., Nooman, Z., et al., (2000): The epidemiology of schistosomiasis in Egypt: summary findings in nine governorates. Am $\mathrm{J}$ Trop Med Hyg; 62: 88-99.

Elsafi, S.H., Al-Maqati, T.N., Hussein, M.I., Adam, A.A., Hassan, M.M., Al Zahrani, E.M. (2013): Comparison of microscopy, rapid immunoassay, and molecular techniques for the detection of Giardia lamblia and Cryptosporidium parvum. Parasitol Res; 112(4): 1641-1646.

Goudal, A., Laude, A., Valot, S., 
Desoubeaux, G., Argy, N., Nourrisson, C., et al., (2019): Rapid diagnostic tests relying on antigen detection from stool as an efficient point of care testing strategy for giardiasis and cryptosporidiosis? Evaluation of a new immunochromatographic duplex assay. Diagn Microbiol Infect Dis; 93(1): 33-36.

Grazioli, B., Matera, G., Laratta, C., Schipani, G., Guarnieri, G., Spiniello, E., et al., (2006): Giardia lamblia infection in patients with irritable bowel syndrome and dyspepsia: a prospective study. World J Gastroenterol; 12(12): 1941-1944.

Gupta, E., Bhalla, P., Khurana, N., Singh, T. (2009): Histopathology for the diagnosis of infectious diseases. Indian J Med Microbiol; 27(2): 100-106.

Hanevik, K., Hausken, T., Morken, M.H., Strand, E.A., Mørch, K., Coll, P., et al., (2007): Persisting symptoms and duodenal inflammation related to Giardia duodenalis infection. J Infect; 55(6): 524-530.

Ikeh, E., Obadofin, M., Brindeiro, Baugher, G., Frost, F. and Vanderjagt, D. (2006): Intestinal parasitism in rural and areas of north central Nigeria: an update. Int J Microbiol; 2(1): 1-12.

Jahani, M., Shafiee, R., Safavi Naeini, P., Rezaian, M., Amini, M., Ebrahimi Daryani, E., et al., (2008): Prevalence of small bowel protozoan among dyspeptic patients who underwent upper gastrointestinal endoscopy (Tehran 2004-2006). JBUMS; 10(1): 60-66.

Johnston, S.P., Ballard, M.M., Beach, M.J., Causer, L., Wilkins, P.P. (2003): Evaluation of three commercial assays for detection of Giardia and Cryptosporidium organisms in fecal specimens. J Clin Microbiol; 41(2): 623-626.

Kerlin, P., Ratnaike, R., Butler, R., Gehling,
N., Grant, A. (1978): Prevalence of giardiasis. Am J Digest Dis; 23(10): 940-942.

Koch, M.A., Zurad, E.G. (2020): Esophagogastroduodenoscopy. Ch. 91. In: Pfenninger and Fowler's Procedures for Primary Care, $4^{\text {th }}$ edition. Fowler, G.C. (editor). Elsevier, Philadelphia, USA, pp.639-655.

Mahdi N., Taha S. (2002): The efficiency of duodenal aspirate in the diagnosis of parasitosis and candidiasis. Qatar Med J; 11(2): 1-3.

Manandhar, U., Karki, S., Shrivastav, S. (2018): A retrospective analysis of parasitic diseases diagnosed by tissue biopsy: a 7-year study. J Institute Med; 40(1): 32-38.

McHenry, R., Bartlett, M.S., Lehman, G.A., O'Connor, K.W. (1987): The yield of routine duodenal aspiration for Giardia lamblia during esophagogastro duodenoscopy. Gastrointest Endosc; 33(6): 425-426.

Mohamed, A.E., Al Karawi, M.A., Ghandour, Z. (1997): Parasitic disease of the liver and biliary tree. Saudi J Gastroenterol; 3(1): 22-28.

Mohamed, A.E., Ghandour, Z.M., Al-Karawi, M.A., Yasawy, M.I., Sammak, B. (2000): Gastrointestinal parasites presentations and histological diagnosis from endoscopic biopsies and surgical specimens. Saudi Med J; 21(7): 629-634.

Nair, K.V., Sharma, M.P., Mithai, S., Tandon, B.N. (1977): Comparative evaluation of diagnostic methods in giardiasis. Indian J Med Res; 66(3): 417-419.

Ngrenngarmlert, W., Lamom, C., Pasuralertsakul, S., Yaicharoen, R., Wongjindanon, N., Sripochang, S., et al., (2007): Intestinal parasitic infections among school children in Thailand. Trop Biomed; 24(2): 83-88.

Pehlivanoğlu, B., DoğanavŞargil, B., Sezak, 
M., Nalbantoğlu, I., Korkmaz, M. (2016): Gastrointestinal parasitosis: Histopathological insights to rare but intriguing lesions of the gastrointestinal tract. Turk Patoloji Derg; 32(2): 82-90.

Regnath, T., Klemm, T., Ignatius, R. (2006): Rapid and accurate detection of Giardia lamblia and Cryptosporidium spp. antigens in human fecal specimens by new commercially available qualitative immunochromatographic assays. Eur $\mathrm{J}$ Clin Microbiol Infect Dis; 25(12): 807-809.

Robert, M., Kliegman, M.D., Bonita, M.D., Stanton, M.D., Joseph, S.T. Geme, M.D., et al., (2016): Cryptosporidium, Isospora, Cyclospora, and Microsporidia. Ch. 283. In: Nelson Textbook of Pediatrics, 20 ${ }^{\text {th }}$ edition. Elsevier Inc., Philadelphia, pp. 1695-1696.

Roberts, W.G., Green, P.H., Ma, J. Carr, M., Ginsberg, A.M. (1989): Prevalence of cryptosporidiosis in patients undergoing endoscopy: evidence for an asymptomatic carrier state. Am J Med; 87(5): 537-539.

Rosenblatt, J.E., Reller, L.B., Weinstein, M.P. (2009): Laboratory diagnosis of infections due to blood and tissue parasites. Clin Infect Dis; 49(7): 1103-1108.

Santos, R.B., Fonseca, L.E., Santana, A.T.,
Silva. C.A., Guedes, J.C. (2011): Clinical, endoscopic and histopathological profiles of parasitic duodenitis cases diagnosed by upper digestive endoscopy. Arq Gastroenterol; 48(4): 225-230.

Wahnschaffe, U., Ignatius, R., Loddenkemper, C., Liesenfeld, O., Muehlen, M., Jelinek, T., et al., (2007): Diagnostic value of endoscopy for the diagnosis of giardiasis and other intestinal diseases in patients with persistent diarrhea from tropical or subtropical areas. Scand J Gastroenterol; 42(3): 391-396.

Washam, M., Frenck, R.W. (2018): Giardia Intestinalis. Ch. 265. In: Principles and practice of pediatric infectious diseases, $5^{\text {th }}$ edition. Long, S., Prober, C., Fischer, M. (editors). Elsevier Inc., Philadelphia, pp. 1317-1321.

Zafar, M.N., Baqai, R., Lodi, T.Z., Ahmad, S., Ahmed, W., Qureshi, H. (1991): Giardia lamblia in patients undergoing upper GI endoscopy. JPMA; 41(4): 74-75.

Zaher, T.I., Emara, M.H., Darweish, E., Abdul-Fattah, M., Bihery, A.S., Refaey, M.M., et al., (2012): Detection of parasites during upper gastrointestinal endoscopic procedures. Afro-Egypt J Infect Endem Dis; 2 (2): 62-68.

\section{How to cite this article:}

Noha Essam Eldin Abd El Rehem Shalaby, Ahmad Ali Othman, Mamdouh Ahmed Gabr and Nabila Abd El Azeem Yassen. 2020. Prevalence of Intestinal Parasitic Infections during Upper Gastrointestinal Endoscopy. Int.J.Curr.Microbiol.App.Sci. 9(07): 2888-2905. doi: https://doi.org/10.20546/ijcmas.2020.907.342 